\title{
Prevention of hepatitis B virus reactivation in lymphoma patients
}

\author{
Blanca Sanchez-Gonzalez ${ }^{1,2 *}$, Montserrat Garcia-Retortillo ${ }^{3}$, Teresa Murcia ${ }^{1}$, Laia Martinez ${ }^{2}$, Ricard Sola ${ }^{3}$, Francesc Garcia-Pallarols ${ }^{1,2}$ and \\ Antonio Salar ${ }^{1,2}$ \\ ${ }^{1}$ Department of Hematology, Hospital del Mar, Barcelona, Spain \\ ${ }^{2}$ IMIM (Hospital del Mar Medical Research Institute), Barcelona, Spain \\ ${ }^{3}$ Liver Section, Department of Gastroenterology, Hospital del Mar, Barcelona, Spain
}

\begin{abstract}
We report a retrospective analysis of lymphoma patients with chronic HBV or resolved HBV treated with chemotherapy, immunotherapy or immunochemotherapy who followed our Institution guidelines to prevent HBV reactivation. The aim of our study was to retrospectively evaluate the management of patients with B-cell lymphoma and $\mathrm{HBV}$ infection receiving chemotherapy or/and rituximab, since the implementation of a consensus algorithm to prevent $\mathrm{HBV}$ reactivation in our centre, focusing on the adherence to the guidelines, $\mathrm{HBV}$ reactivation rate and patient outcome

Current guidelines for management of chronic HBV recommend routine antiviral HBV prophylaxis before chemotherapy and, particularly, with rituximab-containing therapies. In the real world, adherence to these guidelines is scarce. Moreover, there is little evidence-based consensus in the management of patients with resolved HBV infection.

Forty-five out of 227 (19.8\%) B-cell lymphomas had HBV infection. They were categorized patients in three HBV risk groups: group A: active chronic HBV who received HBV treatment; group B: inactive carriers who received HBV prophylaxis; group C: resolved HBV assigned either to antiviral HBV prophylaxis if they received rituximab or to follow-up only if they received rituximab-free chemotherapy. The adherence to our algorithm was $93 \%$. Twenty-five patients (63\%) started antiviral prophylaxis or treatment. Two patients developed HBV reactivation and in both cases reactivation ocurred within the first 6 months after finishing antiviral prophylaxis. In summary, In conclusion, our algorithm strategy efficiently prevents HBV reactivation in B-cell lymphoma patients receiving therapy from an area with high prevalence of HBV infection. Our approach is simple to use, allows a high adherence in the real world and is well-tolerated. We suggest that longer follow-up than actually recommended in current guidelines may be necessary to detect late HBV reactivations.
\end{abstract}

\begin{abstract}
Abbreviations: DLBCL: diffuse large B-cell lymphoma; ETV: entecavir; FL: follicular lymphoma; HBV: Hepatitis B virus; HIV: Human immunodeficiency virus; HCV: Hepatitis C virus; HL: Hodgkin's lymphoma; HSCT: hematopoietic stem cells transplantation; IPI: International Prognostic Index; IQR: Interquartile Range; M: male; MCL: mantle-cell lymphoma; NP: no prophylaxis; R: rituximab; SD: standard deviation; TDF: tenofovir; 3TC: lamivudine.
\end{abstract}

\section{Introduction}

Chemotherapy-induced hepatitis B virus (HBV) reactivation is a well-recognized complication and is a potentially life-threatening condition in cancer patients with chronic HBV (hepatitis B surface antigen [HBsAg]-positive) [1]. Rituximab, an anti-CD20 monoclonal antibody, has been associated with an increased risk of $\mathrm{HBV}$ reactivation in chronic HBV patients and even in those with resolved infection (HBsAg negative/hepatitis B core antibody [anti-HBc]-positive) [2], even though the reported frequency varies among different studies (44\% for chronic HBV and 25\% for resolved infection) [3,4].

Current guidelines for management of chronic HBV recommend routine antiviral HBV prophylaxis before chemotherapy and, particularly, with rituximab-containing therapies [5]. In the real world, adherence to these guidelines is scarce. Moreover, there is little evidence-based consensus in the management of patients with resolved HBV infection. The aim of our study was to restrospectively evaluate the management of patients with B-cell lymphoma and HBV infection receiving chemotherapy or/and rituximab, since the implementation of a consensus algorithm to prevent $\mathrm{HBV}$ reactivation in our center, focusing on the adherence to the guidelines, HBV reactivation rate and patient outcome.

\section{Patients and methods}

\section{Study design}

From January 2007 to December 2015, all patients with lymphoma were screened for human immunodeficiency virus (HIV), hepatitis $\mathrm{C}$ virus (HCV) and $\mathrm{HBV}$ (HBsAg and anti-HBc) before therapy. In patients carrying any of these HBV markers, serum HBV DNA levels were tested and subsequently, were assigned in three groups. Group A: active chronic HBV who received HBV treatment; group B: inactive carriers who received HBV prophylaxis; group C: resolved HBV assigned either to antiviral HBV prophylaxis if they received rituximab or to follow-up only if they received rituximab-free chemotherapy. HBV antiviral treatment/prophylaxis types were lamivudine, entecavir

${ }^{\star}$ Correspondence to: Blanca Sanchez Gonzalez, MD, .Department of Hematology, Hospital del Mar, Barcelona, Spain, Tel: (034)93 248 3343; Fax: (034)93 248 3343; E-mail: 97894@parcdesalutmar.cat

Key words: hepatitis B virus reactivation, lymphoma, rituximab, chemotherapy Received: July 04, 2019; Accepted: July 19, 2019; Published: July 22, 2019 
or tenofovir. In the HBV antiviral prophylaxis/treatment group, therapy was initiated with 1 week before the first course of chemotherapy and continued until 6-12 months after completing therapy. The study was approved by the institutional board at the Parc de Salut Mar.

\section{Definitions}

HBV viral status definitions: Active chronic HBV infection: $\mathrm{HBsAg}$ positive, anti-HBc positive and $\mathrm{HBV}$ DNA >2000 IU/mL; inactive carriers: HBsAg positive, Anti-HBc positive, HBV DNA undetectable or $<2000 \mathrm{IU} / \mathrm{mL}$ with normal transaminases; resolved $\mathrm{HBV}$ : HBsAg negative, anti-HBc positive, HBV DNA undetectable. $\mathrm{HBV}$ reactivation was defined as an increase in serum HBV DNA $(\geq 1$ $\log 10$ ), regardless of liver biochemistry or HBsAg status. Delayed HBV reactivation was defined as $\mathrm{HBV}$ reactivation ocurring at least three months after ending antiviral HBV prophylaxis. Hepatitis flare was defined as serum ALT level more than $100 \mathrm{IU} / \mathrm{L}$ and was attributed to $\mathrm{HBV}$ reactivation if it was preceded or accompanied by detectable DNA HBV or by the reappearance of HBsAg in serum in patiens with resolved HBV infection.

\section{Outcomes and Follow-up}

All patients were monitored at baseline, every 3 months during therapy and until 24 months after finishing therapy. Assessments performed included a liver biochemistry panel, serum HBV DNA, HBsAg and anti-HBs levels.

\section{Statistical Methods}

Statistical comparisons were made using the chi-square test or Fisher's exact test, as appropriate. Cumulative HBV reactivation rate was calculated using Kaplan-Meier curves (SPSS v17.0; SPSS, Chicago, IL).

\section{Results}

\section{Patient Disposition and Characteristics}

Two hundred and twenty seven lymphoma patients received chemotherapy or immunochemotherapy, $63 \%$ of them received rituximab alone or in combination with chemotherapy. Fourty five patients had some HBV test positive and 43 were enrolled onto our study. One patient was assigned to group A, 3 to group B and 39 to group C. Fourteen patients $(6 \%)$ had coinfection with hepatitis $C$ virus and 12 patients (5\%) coinfection with HIV. HBV guideline adherence was $93 \%$. Antiviral treatment/prophylaxis was administered in all patients in groups A and B and in 23 patients (70\%) in the group C (13 underwent only follow-up) (Table 1). Three patients presented intolerance to tenofovir and were switched to lamivudine. None patient stopped antiviral prophylaxis because of related-adverse events.

Table 1. Baseline Characteristics of Patients

\begin{tabular}{|c|c|c|c|c|c|c|c|}
\hline \multicolumn{4}{|l|}{ Group B $(n=3)$} & \multicolumn{4}{|c|}{ Group C $(n=36)$} \\
\hline \multirow[b]{2}{*}{ Variables } & & \multirow[b]{2}{*}{ No. of Patients } & \multirow[b]{2}{*}{$\%$} & \multicolumn{2}{|c|}{$\begin{array}{l}\text { Rituximab +/- Chemotherapy } \\
(\mathrm{n}=23)\end{array}$} & \multicolumn{2}{|c|}{$\begin{array}{l}\text { Rituximab-free chemotherapy } \\
(\mathrm{n}=13)\end{array}$} \\
\hline & & & & No. of Patients & $\%$ & No. of Patients & $\%$ \\
\hline Age (years) & $\begin{array}{l}\text { Median } \\
\text { IQR }\end{array}$ & \multicolumn{2}{|l|}{$\begin{array}{l}44.9 \\
38.4-50\end{array}$} & \multicolumn{2}{|l|}{$\begin{array}{l}72.1 \\
58-77\end{array}$} & \multicolumn{2}{|l|}{$\begin{array}{l}55.3 \\
43.2-63.5\end{array}$} \\
\hline \multirow{2}{*}{ Sex } & Men & 2 & 66.7 & 14 & 60.9 & 11 & 84.6 \\
\hline & Female & 1 & 33.3 & 9 & 39.1 & 2 & 15.4 \\
\hline \multirow{5}{*}{ Disease } & DLBCL & 0 & 0 & 13 & 56.5 & 1 & 7.7 \\
\hline & FL & 0 & 0 & 4 & 17.4 & 0 & 0 \\
\hline & MCL & 0 & 0 & 1 & 4.3 & 0 & 0 \\
\hline & HL & 1 & 33.3 & 0 & 0 & 9 & 69.2 \\
\hline & Other* & 1 & 66.7 & 5 & 21.8 & 3 & 23.1 \\
\hline \multirow{4}{*}{ IPI } & Low Risk & 1 & 33.3 & 6 & 26.1 & 7 & 53.8 \\
\hline & Low-intermediate Risk & 2 & 66.7 & 6 & 26.1 & 5 & 38.5 \\
\hline & High-intermediate Risk & 0 & 0 & 8 & 34.8 & 1 & 7.7 \\
\hline & High Risk & 0 & 0 & 3 & 13 & 0 & 0 \\
\hline \multirow{2}{*}{ Coinfection } & $\mathrm{HCV}$ & 1 & 33.3 & 4 & 17.4 & 0 & 0 \\
\hline & HIV & 0 & 0 & 4 & 17.4 & 1 & 7.7 \\
\hline \multirow{2}{*}{ Baseline ALT (UI/L) } & Mean & 17.3 & & \multicolumn{2}{|l|}{18.1} & \multicolumn{2}{|l|}{19.2} \\
\hline & SD & 6.5 & & \multicolumn{2}{|l|}{11.4} & \multicolumn{2}{|l|}{6.8} \\
\hline \multirow{4}{*}{ Prophylaxis Drugs } & No prophylaxis & 0 & 0 & 5 & 21.7 & 10 & 76.9 \\
\hline & ETV & 1 & 33.3 & 7 & 30.4 & 0 & 0 \\
\hline & TDF & 2 & 66.7 & 8 & 34.8 & 2 & 15.4 \\
\hline & $3 \mathrm{TC}$ & 0 & 0 & 3 & 13 & 1 & 7.7 \\
\hline \multirow[t]{2}{*}{$\begin{array}{l}\text { Duration of prophylaxis } \\
\text { treatment (months) }\end{array}$} & Median & \multicolumn{2}{|l|}{42.6} & \multicolumn{2}{|l|}{13.4} & \multicolumn{2}{|l|}{7.4} \\
\hline & IQR & \multicolumn{2}{|l|}{$13-46.3$} & \multicolumn{2}{|l|}{$9.6-17.4$} & \multicolumn{2}{|l|}{$5.3-21.6$} \\
\hline Duration of follow-up (months) & Median & 42.6 & & 17.9 & & 13.9 & \\
\hline & IQR & $16.9-46.3$ & & $9.7-26.8$ & & $5.5-21.8$ & \\
\hline Status at the end of the study & Follow-up & 3 & 100 & 14 & 60.9 & 2 & 15.4 \\
\hline & Discharge & 0 & 0 & 5 & 21.7 & 8 & 61.5 \\
\hline & Death of any cause & 0 & 0 & 0 & 0 & 3 & 23.1 \\
\hline & Lose of follow up & 0 & 0 & 3 & 13 & 0 & 0 \\
\hline $\begin{array}{l}\text { Abbreviations: DLBCL: diffuse } \\
\text { lymphoma; IQR: Interquartile Ra } \\
\text { "Other: mucosa-associated lympl }\end{array}$ & $\begin{array}{l}\text { large B-cell lymphoma; } \\
\text { ange; IPI: International Pr } \\
\text { hoid tissue lymphoma, sm }\end{array}$ & $\begin{array}{l}\text { TV: entecavir; FL } \\
\text { gnostic Index; } \mathrm{MC}\end{array}$ & $\begin{array}{l}\text { llicula } \\
\text { mantl }\end{array}$ & $\begin{array}{l}\text { CV: hepatitis C } \\
\text { a; SD: standard }\end{array}$ & $\begin{array}{l}\text {; HIV: huma } \\
\text { ation; TDF: }\end{array}$ & $\begin{array}{l}\text { nodeficiency viru } \\
\text {; 3TC: lamivudin }\end{array}$ & IL: Hodgkin' \\
\hline
\end{tabular}




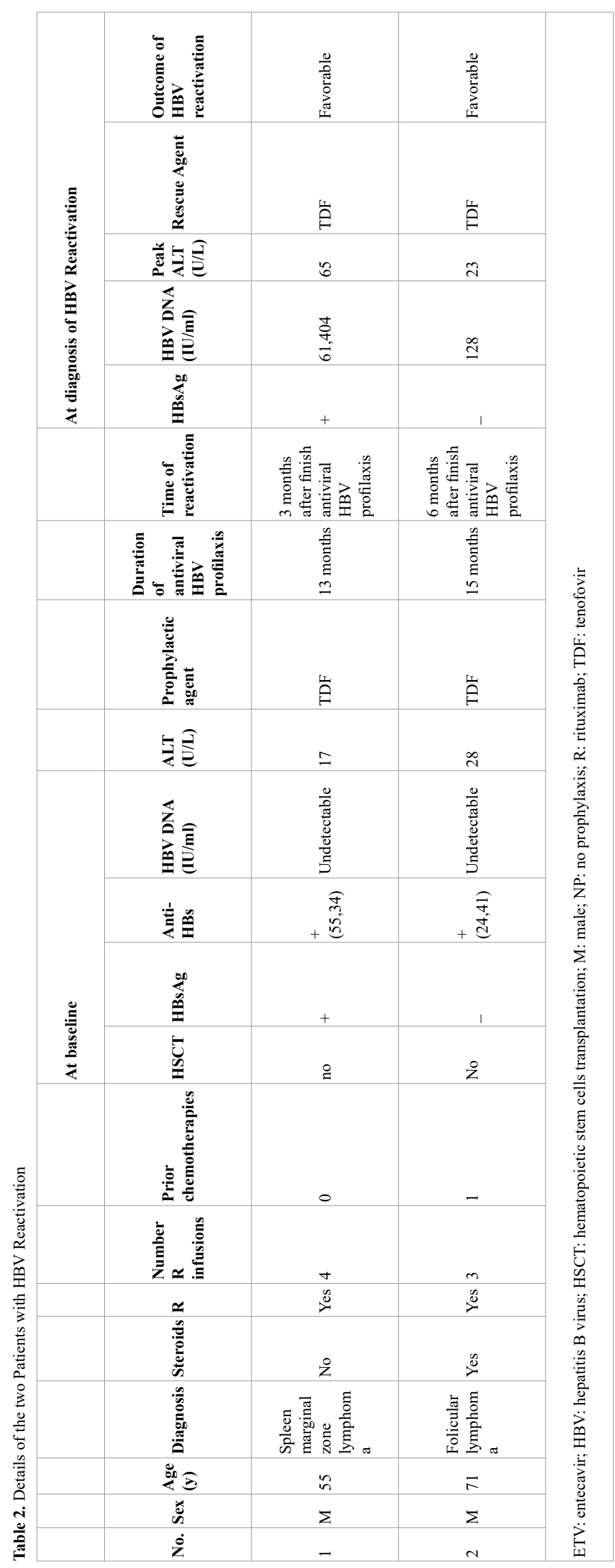

\section{Incidence of HBV Reactivation}

With a median follow-up of 21 months, 2 patients developed HBV reactivation during lymphoma treatment (one from group $B$ and one from group $\mathrm{C}$ ). Both patients received rituximab based-treatment and both developed HBV reactivation within the first 6 months after finishing antiviral HBV profilaxis (delayed HBV reactivation), none of them had hepatitis flare and the outcome was favourable (Table 2). Cumulative incidence of $\mathrm{HBV}$ reactivation at 12 and 24 months were $0 \%$ and $9 \%$, respectively.

\section{Discussion}

Chronic HBV infection is a worldwide public health problem. Spain is a country with intermediate prevalence $(2-8 \% \mathrm{HbsAg}$ positive), as others Mediterranean countries, although recent studies have showed lower rates $(0.32-0.37 \% \mathrm{HbsAg}$ positive) [6]. We have observed a prevalence of anti-HBc of $14 \%$ in patients with B-cell lymphoma, which is higher than the prevalence seen in the general population (8.7\%) [6]. Other recent studies have also reported a high prevalence of anti-HBc in Asian patients with lymphoma $(26.5 \%$ and 44.2\%) [3-4]. The association between HBV infection and B-cell lymphomas is not as well established as the etiopathogenic role of $\mathrm{HCV}$ in some lymphoproliferative conditions. However, it has recently been described that patients with HBV have almost double the risk of developing a non-Hodgkin lymphoma [7]. The high prevalence of anti$\mathrm{HBc}$ positive in our patients with B-cell lymphoma could be explained by this hypothesis and also by the high-risk life-style characteristics of part of the referral population of our center.

Standard treatment of CD20+ B-cell lymphoma patients includes an anti-CD20 monoclonal antibody, such as rituximab, that increases the risk of reactivation of $\mathrm{HBV}$ [2]. Given the high prevalence of HBV infection in patients with lymphoma, there is a need to consider management strategies for these patients during treatment. In order to avoid HBV reactivations, several studies have demonstrated the usefulness of HBV antiviral prophylaxis for patients who receive treatment with rituximab or other anti-CD20 monoclonal antibodies $[8,9]$. Although current guidelines for management of chronic HBV recommend routine antiviral prophylaxis, but there is little evidencebased consensus in resolved HBV. In our study $10 \%$ of the patients had active chronic HBV or were inactive carriers and all of them received $\mathrm{HBV}$ antiviral drugs previous therapy. The remaining $90 \%$ patients had resolved $\mathrm{HBV}$ and $58 \%$ of them initiated prophylaxis with tenofovir or entecavir. Only three cases had intolerance to tenofovir and they were switched to lamivudine, a treatment which is associated with higher rate of HBV resistance development but that might be a reasonable option for intolerant cases.

We have observed a high adherence (93\%) of our algorithm, probably related to its easy applicability, the motivation of the multidisciplinary research team and their awareness of the importance of HBV reactivation prevention. Moreover, our HBV reactivation rate was only $5 \%$, a figure which is lower than that described in previous studies (17.9\%) in patients without HBV antiviral prophylaxis [9]. Therefore, our algorithm for the management of HBV, together with its high compliance, translates into an evident clinical benefit in terms of reduction of the reactivation rate and its potential severe complications. Another remarkable finding of our study was the good safety profile of antiviral drugs in patients receiving cytostatic treatment.

Another relevant finding was that our two cases with delayed HBV reactivation were detected at 3 and 6 months after ending antiviral 
prophylaxis. Current recommendations in the consensus guidelines for the management of these patients suggest only 12 months of antiviral prophylaxis after the end of the antineoplastic treatment [5]. A recent study showed that the median time from the end of HBV prophylaxis to reactivation was 2.9 months [10]. This observation and our experience suggest that only 12 months of prophylaxis might be insufficient and do not confer adequate $\mathrm{HBV}$ protection, especially in the context of immunosuppressed patients by their disease and/or their treatments.

The limitations of our study include sample size and, fortunately, the low number of reactivations. Both circumstances prevented analysis of risk factors involved in the HBV reactivation. In addition, inclusion of different subtypes of B-cell lymphomas, lymphomas at different stages and several schedules of therapy could have influenced the risk of HBV reactivation as well as the need for receiving prophylaxis.

In conclusion, our algorithm strategy efficiently prevents HBV reactivation in B-cell lymphoma patients receiving therapy from an area with high prevalence of HBV infection. Our approach is simple to use, allows a high adherence in the real world and is well-tolerated. We suggest that longer follow-up than actually recommended in current guidelines may be necessary to detect late HBV reactivations. Consequently, larger validation studies are needed to confirm our data and to establish risk factors for HBV reactivation, as well as the optimal antiviral prophylaxis, especially in the context of new available inmunomodulatory agents for lymphoma treatment.

\section{Acknowledgments}

None

\section{Funding}

This study has not been funded.

\section{Availabily of data and materials}

The datasets used and/or analyzed during the current study are available from the corresponding author on reasonable request.

\section{Authors' contributions}

BSG, MGR, TR, EG, RS, and AS interpreted the study data and contributed to writing the manuscript. BSG, FGP, TR and AS performed the data. All authors read and approved the final manuscript.

\section{Ethics approval and consent to participate}

This study was conducted in full conformance with the ICH E6 Guideline for Good Clinical Practice and the principles of the Declaration of Helsinki.
It was approved by the institutional review boards or ethics committees of the study sites.

\section{Consent for publication}

Not applicable.

\section{Competing interests}

Blanca Sanchez-Gonzalez reports noninstitutional research funding and is a consultant and/or speaker bureau for Novartis, Amgen, Alexion, Gilead and Shire.

Antonio Salar reports institutional research funding from Roche, and is a consultant and/or speaker bureau for Roche, Gilead, Janssen and Celgene.

The others co-authors declare no conflict of interest

\section{References}

1. Liang R, Lau GK, Kwong YL (1999) Chemotherapy and bone marrow transplantation for cancer patients who are also chronic hepatitis B carriers: a review of the problem. $J$ Clin Oncol 17: 394-398. [Crossref]

2. Evens AM, Jovanovic BD, Su YC, Raisch DW, Ganger D, et al. (2011) Rituximabassociated hepatitis B virus (HBV) reactivation in lymphoproliferative diseases: metaanalysis and examination of FDA safety reports. Ann Oncol 22: 1170-1180.

3. Yeo W, Chan TC, Leung NW, Lam WY, Mo FK, et al. (2009) Hepatitis B virus reactivation in lymphoma patients with prior resolved hepatitis B undergoing anticancer therapy with or without rituximab. J Clin Oncol 27: 605-611.

4. Seto WK, Chan TS, Hwang YY, Wong DK, Fung J, et al. (2014) Hepatitis B reactivation in patients with previous hepatitis B virus exposure undergoing rituximab-containing chemotherapy for lymphoma: a prospective study. J Clin Oncol 32: 3736-43.

5. EASL (2012) European Association for The Study Of The Liver. EASL clinical practice guidelines: Management of chronic hepatitis B virus infection. J Hepatol 57: 167-185.

6. Schweitzer A, Horn J, Mikolajczyk RT, Krause G, Ott JJ (2015) Estimations of worldwide prevalence of chronic hepatitis B virus infection: a systematic review of data published between 1965 and 2013. Lancet 386: 1546-1555.

7. Engels EA, Cho ER, Jee SH (2010) Hepatitis B virus infection and risk of non-Hodgkin lymphoma in South Korea: a cohort study. Lancet Oncol 11: 827-834.

8. Huang YH, Hsiao LT, Hong YC, Chiou TJ, Yu YB, et al. (2013) Randomized controlled trial of entecavir prophylaxis for rituximab-associated hepatitis B virus reactivation in patients with lymphoma and resolved hepatitis B. J Clin Oncol 31: 2765-2772.

9. Huang H, Li X, Zhu J, Ye S, Zhang H (2014) Entecavir vs lamivudine for prevention of hepatitis B virus reactivation among patients with untreated diffuse large B-cell lymphoma receiving R-CHOP chemotherapy: a randomized clinical trial. JAMA 312: 2521-2530. [Crossref]

10. Liu WP, Wang XP, Zheng W, Ping LY, Zhang C, et al. (2016) Hepatitis B virus reactivation after withdrawal of prophylactic antiviral therapy in patients with diffuse large B cell lymphoma. Leuk Lymphoma 57: 1355-1362. 